\title{
Negative-Ion/Positive-Ion Coincidence Yields of Core-Excited Water
}

\author{
Christian Stråhlman, ${ }^{*} \dagger$ Antti Kivimäki, ${ }^{\ddagger}$ Robert Richter, ${ }^{\top}$ and Rami Sankari ${ }^{\dagger}$ \\ $\dagger$ †AX IV Laboratory, Lund University, P.O. Box 118, 22100 Lund, Sweden \\ $\ddagger$ Consiglio Nazionale delle Ricerche - Istituto Officina dei Materiali, Laboratorio TASC, \\ 34149 Trieste, Italy \\ 9Elettra-Sincrotrone Trieste, Area Science Park, 34149 Trieste, Italy \\ E-mail: Christian.Strahlman@maxiv.lu.se
}

Phone: +46462220000 


\begin{abstract}
We report yields of mass-resolved negative ions and positive ions measured in coincidence after core-excitation of water molecules. The analysis of negative-ion/positiveion and negative-ion/positive-ion/positive-ion coincidence events provides new information on pathways leading to negative ion production, enhancing the present understanding of the dissociation processes of the water molecule. Dissociation following (resonant) Auger decay dominates negative ion production, but radiative decay is shown to contribute above the $\mathrm{O}$ 1s ionization threshold. A peak in the $\mathrm{H}^{-} / \mathrm{O}^{+}$yield above the $\mathrm{O}$ 1s threshold is attributed to decay from doubly excited states.
\end{abstract}

\title{
Introduction
}

The scientific study of water has deep historical roots. The natural philosophers of ancient Greece, more than two millennia ago, named water one of the four classical elements; funda-

mental and indivisible. While water still should be regarded as fundamental, supporting all known forms of life, it is not indivisible. Studies of the fragmentation of the water molecule have provided deep insights into the constitution and chemical properties of this remarkable element. In contrast to the apparent simplicity of the molecule, the chemistry of liquid and solid water is very complex and is yet not fully understood. ${ }^{1,2}$

Photoexcitation of the $\mathrm{H}_{2} \mathrm{O}$ molecule can induce a multitude of electronic processes, some of which lead to dissociation and fragmentation of the molecule. Dissociation can follow either core-excitation to unoccupied orbitals or core-ionization (i.e. removal of a core electron), but also excitation and ionization processes in the valence region, provided that the photon energy exceeds the dissociation limit. The core-excited states of the $\mathrm{H}_{2} \mathrm{O}$ molecule typically relax on the fs time-scale by emission of a (resonant) Auger electron ${ }^{3}$ or, with much lower probability, a fluorescent photon, ${ }^{4,5}$ which is a competing process. Within this time-scale, the relaxation is considered to precede dissociation (i.e. separation of the nuclei). The then valence-excited molecules can subsequently dissociate. An exception from 
this model is ultra-fast dissociation, shown to occur at the $\mathrm{O} 1 s \rightarrow 4 a_{1}$ resonance, ${ }^{6}$ where the core-excited $\mathrm{H}_{2} \mathrm{O}^{* *}$ molecule dissociates to a $\mathrm{OH}^{* *} / \mathrm{H}$ pair before electronic decay (** denotes a core-excited species). In all cases, dissociation can produce positive ions, neutral fragments, and, with much lower probability, negative ions. Piancastelli et al. ${ }^{7}$ measured positive-ion yields (PIY) and positive-ion coincidence spectra (PIPICO) of water with up to three ionic fragments, while Stolte et al. ${ }^{8}$ have measured negative ion yields (NIY) in the same energy region. PIYs and NIYs of water have also been measured with high resolution in the valence region. ${ }^{9,10}$

Negative-ion production has received much less attention than that of positive ions. Partly this is due to the experimental difficulty to separate negative ions from electrons, which are always present in large abundance when molecules are probed with soft x-rays. In addition, negative ion production is a weak fragmentation channel. This, on the other hand, makes it a sensitive probe for fragmentation studies. While negative ions have been observed at the core edges of several small molecules, ${ }^{8,11-18}$ studies of negative ions in coincidence with other fragments are rare. Rühl and Flesch ${ }^{19}$ used a setup consisting of two identical time-offlight spectrometers for negative and positive ion detection to study the production of $\mathrm{O}^{-}$in coincidence with $\mathrm{C}^{+}$and $\mathrm{O}^{+}$at the $\mathrm{C} 1 s \rightarrow \pi^{*}$ resonance in $\mathrm{CO}_{2}$ and confirmed the existence of a three-body fragmentation channel $\mathrm{O}^{-} / \mathrm{C}^{+} / \mathrm{O}^{+}$. As $\mathrm{O}^{-}$was the only observed negative species and showed enhanced yield only at one resonance at the $\mathrm{C}$ 1s edge, the comparison of fragmentation patterns against other excited states was not possible.

We report here measurements of the production of negative and positive ions from $\mathrm{H}_{2} \mathrm{O}$ at the photon energy region close to the $\mathrm{O} 1 \mathrm{~s}$ ionization potential (IP) at $539.9 \mathrm{eV}$. Our study complements earlier fragmentation studies of $\mathrm{H}_{2} \mathrm{O}$ at the $\mathrm{O} 1 s$ edge. ${ }^{7,8}$ We have developed an experimental setup with two time-of-flight spectrometers working in tandem and a momentum filter for deflecting electrons. ${ }^{20}$ With this instrument all ionic fragments can be mass-resolved and complete fragmentation pathways involving one negative ion and several positive ions can be charted. For $\mathrm{H}_{2} \mathrm{O}$, this implies double (NIPICO, negative-ion/positive- 
ion coincidence) and triple (NIPIPICO, negative-ion/positive-ion/positive-ion coincidence) ionic detection. Charting the negative-ion fragmentation provides deeper insight to molecular processes, non-accessible by positive ion coincidence yields alone.

\section{Experiment}

The experiments were performed at the Gas Phase Photoemission beamline of the Elettra synchrotron radiation laboratory (Trieste, Italy). The beamline has been described in detail before. ${ }^{21,22}$ An undulator provides light and a spherical grating monochromator is used to select the photon energy. The photon energy range of the beamline is $13.5-900 \mathrm{eV}$. Five interchangeable gratings ensures that high resolving power $\left(>10^{4}\right)$ can be achieved.

An effusive jet of $\mathrm{H}_{2} \mathrm{O}$ vapor was let into the chamber through a gas needle controlled by a leak valve. The valve was heated to $50^{\circ} \mathrm{C}$ to prevent condensation of the vapor. The position of the needle was adjusted so that the photon beam crossed the molecular beam in the interaction region of the instrument. When the gas was introduced to the chamber, the pressure increased from the $3 \cdot 10^{-8}$ mbar base pressure to $6.5 \cdot 10^{-7} \mathrm{mbar}$, but it was estimated to be 10-50 times higher in the interaction region. The intensity of the light was monitored with a photodiode at the downstream exit of the chamber. All measured data were normalized to the diode current.

The negative-ion/positive-ion coincidence spectrometer has been described in detail in Ref. ${ }^{20}$ In brief, it consists of two time-of-fight ion spectrometers operating in tandem; one for positive ions and the other for negative ions. The opening angles of the spectrometers' acceptance cones are a function of the kinetic energy distribution of the emitted fragments. Fragments with high kinetic energies, notably $\mathrm{H}^{+}$created from strongly repulsive ionized states, will escape detection in the positive-ion spectrometer to a higher extent than slower fragments. With present voltage settings, the positive ion spectrometer acceptance is complete ( $4 \pi$ solid angle) below $2 \mathrm{eV}$, but drops quickly above that. For this reason, and because 
the complete kinetic energy release (KER) in the fragmentation channels is not known, no quantitative conclusions should be drawn from the measured NIPICO and NIPIPICO yields. Both the spectrometers are installed with their detection axes at the magic angle (54.7 degrees) with respect to the electric vector of the linearly polarized incident radiation. This mounting ensures that the detected intensity is independent of the molecular anisotropy parameter $\beta$, irrespective of the opening angle of the instrument's acceptance cone. ${ }^{23}$

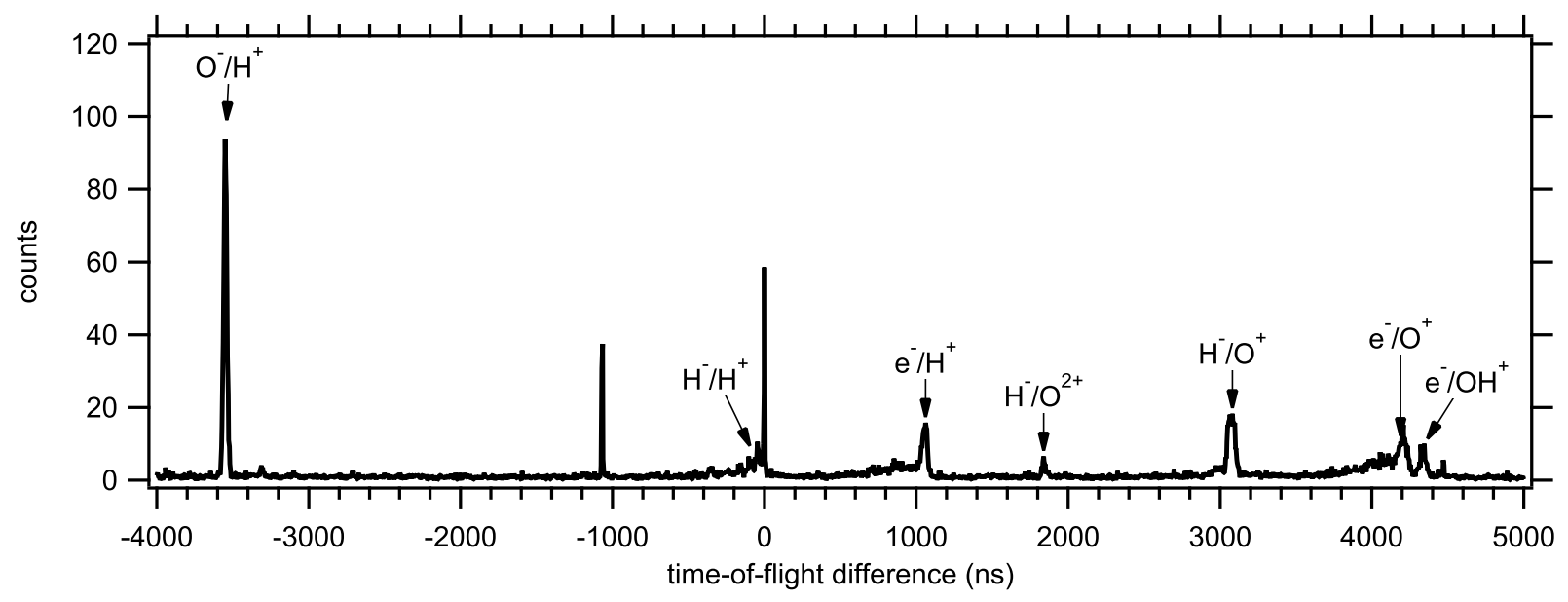

Figure 1: NIPICO TOF spectrum recorded for 40 minutes at the $\mathrm{O} 1 s \rightarrow 2 b_{2}$ resonance (535.8 eV) with 4 ns binning and assignments of the major NIPICO and PEPICO channels. The sharp features at $\Delta t=0 \mathrm{~ns}$ and $\Delta t=-1100 \mathrm{~ns}$ are artifacts arising from hardware (see Ref. ${ }^{20}$ for details). The $\mathrm{H}^{-} / \mathrm{H}^{+}$peak is situated just to the left of the $\Delta t=0$ ns feature.

The negative-ion spectrometer is equipped with a magnetic momentum filter for deflection of electrons. Negative and positive ions are detected by two single anode MCP detectors; the respective signals are amplified and, after passing through a discriminator, read by a time-to-digital converter (TDC). The data in this paper were acquired in the so-called constant extraction mode with continuous triggering. ${ }^{20}$ This acquisition mode was created to allow for recording of coincidences between one negative and several positive ions. Time-stamps from the two detectors were recorded independently during $80 \mathrm{~ms}$ acquisition windows, followed by $20 \mathrm{~ms}$ readout interruptions. In the subsequent computer analysis, positive ion signals recorded within a $22 \mu$ s time--window surrounding a negative ion signal were considered to be coincident, and the differences in their arrival times were stored in a 
data array. If two positive ions arrived within the specified time--window, a NIPIPICO event was recorded.

\section{Results}

Figure 1 shows a typical NIPICO TOF spectrum acquired at the $\mathrm{O} 1 s \rightarrow 2 b_{2}$ resonance (535.8 eV). Aided by simulations of flight times for all possible negative and positive ionic fragments, the peaks can be assigned to coincidences between specific negative ions (or electrons) and specific positive ions. Four NIPICO channels $-\mathrm{O}^{-} / \mathrm{H}^{+}, \mathrm{H}^{-} / \mathrm{H}^{+}, \mathrm{H}^{-} / \mathrm{O}^{+}$and $\mathrm{H}^{-} / \mathrm{O}^{2+}$ - have been observed. In addition, some photoelectron/positive-ion coincidence (PEPICO) channels created by electrons escaping the filter are present. These will not be considered further.

Coincidence yields were measured by scanning three adjacent photon energy regions close to the $\mathrm{O}$ 1s IP. Figures 2 and 3 show NIPICO and NIPIPICO yields in the energy region 532.5-542.0 eV, including the $\mathrm{O} 1 s \rightarrow 4 a_{1}, 2 b_{2}$ and $2 b_{1}$ resonances and the $\mathrm{O} 1$ s ionization threshold. Extra precautions have been taken for the $\mathrm{H}^{-} / \mathrm{H}^{+}$yield. This TOF peak lies very close to the sharp feature at $\Delta t=0 \mathrm{~ns}$, attributed to spurious crosstalk between the two detectors. For some data points, this crosstalk was much increased and time-broadened, causing interference with the $\mathrm{H}^{-} / \mathrm{H}^{+}$peak. These points could however be readily identified and deleted from the $\mathrm{H}^{-} / \mathrm{H}^{+}$yield. No other NIPICO or NIPIPICO yields were affected by the crosstalk.

For the lower photon energy range (532.5-539.0 eV, blue crosses), the photon energy was scanned in $\sim 0.2 \mathrm{eV}$ steps, with four repetitions. Each point was measured for 600 seconds. In this acquisition, the energy steps provided by the monochromator were slightly fluctuating; thereby introducing unequal spacing between the photon energy positions and preventing us from summing the results of different repetitions. Fitting of the three lowest resonance peaks was performed as follows: Three exponentially modified gaussian peaks (i.e. a Gaussian 


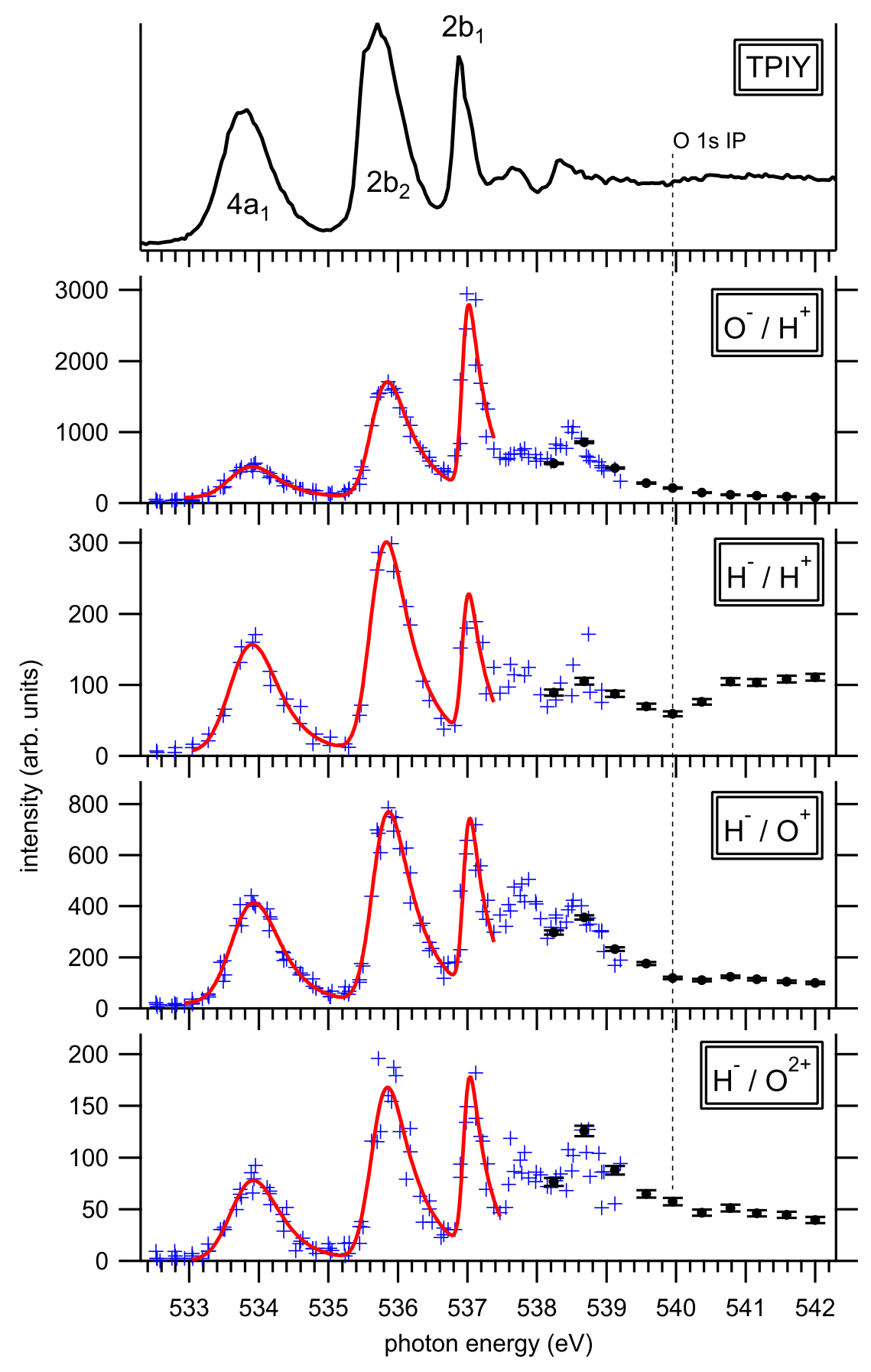

Figure 2: NIPICO yields measured at the $\mathrm{O} 1 \mathrm{~s}$ edge of $\mathrm{H}_{2} \mathrm{O}$. Data points below the $\mathrm{O}$ 1s IP (blue crosses) were collected for 10 minutes each, while the higher energy yields (black circles) were collected for 40 minutes each. Below-IP data has been fitted using the procedure described in the text. The statistical errors are indicated for the higher energies. The total positive ion yield (TPIY) is given for comparison. 


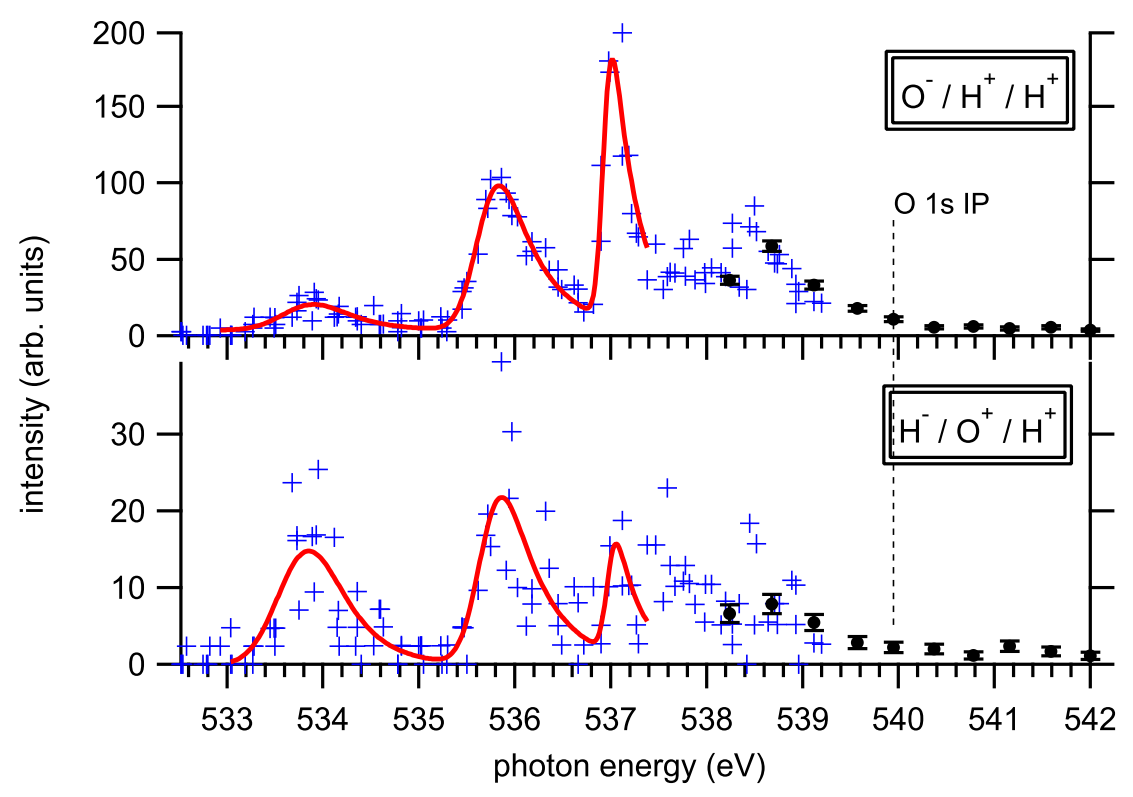

Figure 3: NIPIPICO yields measured at the $\mathrm{O} 1 \mathrm{~s}$ edge of $\mathrm{H}_{2} \mathrm{O}$. Data points below the O 1s IP (blue crosses) were collected for 10 minutes each, while the higher energy yields (black circles) were collected for 40 minutes each. Below-IP data has been fitted using the procedure described in the text. The statistical errors are indicated for the higher energies.

distribution with an independent exponential component) were fitted to the TPIY spectrum in Figure 2. It was assumed that the NIPICO and NIPIPICO peaks had the same shape as the TPIY peaks; NIYs measured by Stolte et al. ${ }^{8}$ have not revealed any large deviations from TPIY peak shapes. The same exponentially modified gaussians were used as trial functions for the fitting of the NIPICO and NIPIPICO yields; the peak shape and position along the energy axis were fixed while the amplitude was variable.

The higher photon energy range $(538.2-542.0 \mathrm{eV}$, black circles) was scanned in $\sim 0.4 \mathrm{eV}$ steps, with three repetitions. Each point was measured for 800 seconds and the three scans were summed. The above-IP NIPICO yields in Figure 4 were measured in $2 \mathrm{eV}$ steps, 50 minutes per energy point acquired in three repetitions. 


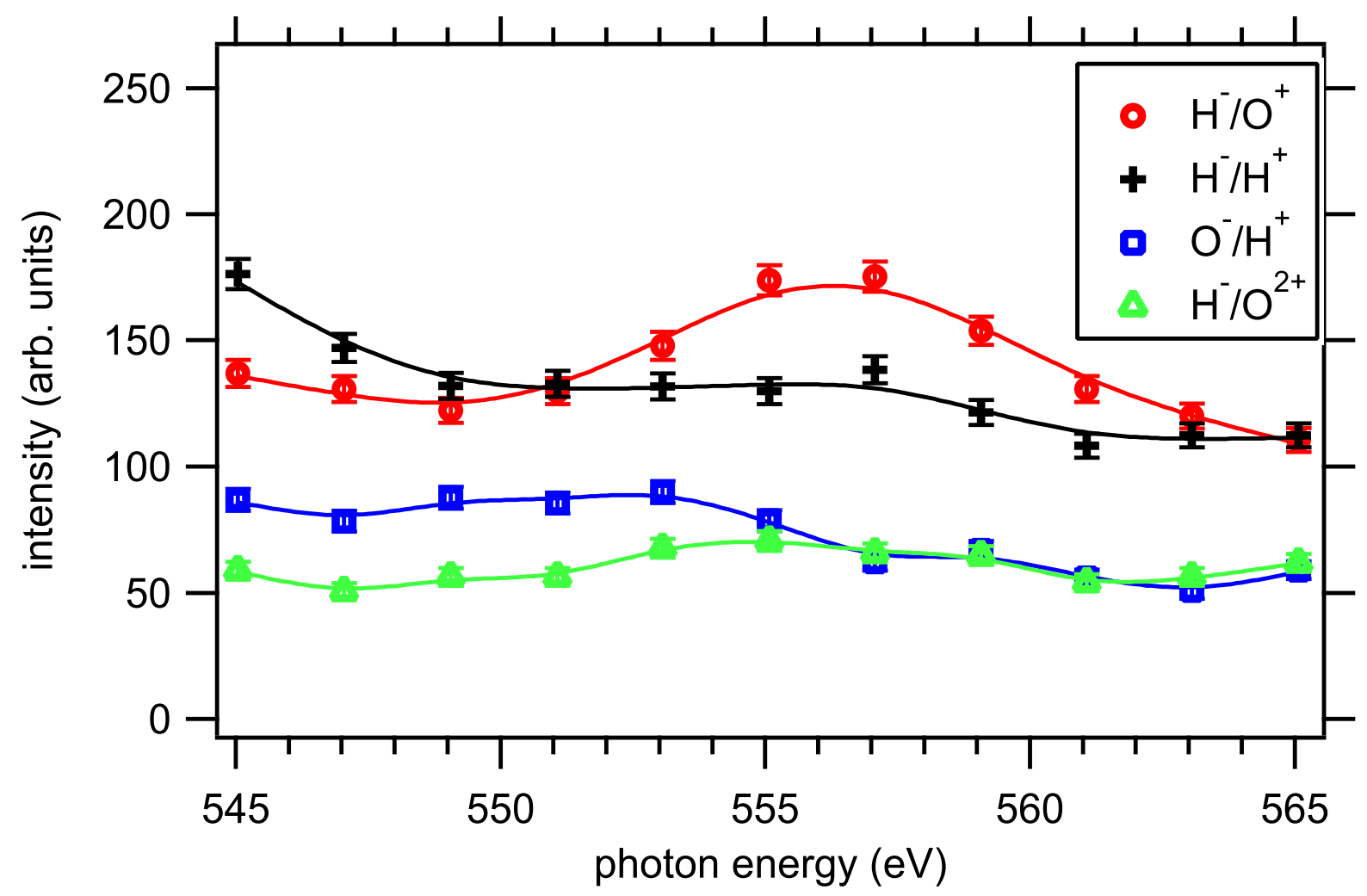

Figure 4: NIPICO yields measured above the $\mathrm{O} 1 \mathrm{~s}$ edge of $\mathrm{H}_{2} \mathrm{O}$. Each data point was measured for 50 minutes. A smoothing spline interpolation has been added. 


\section{Discussion}

All possible fragmentation pathways of $\mathrm{H}_{2} \mathrm{O}$ can be classified by the charge of the parent $\mathrm{H}_{2} \mathrm{O}$ ion before fragmentation. ${ }^{7,8}$ The total number of fragmentation pathways leading to a negative ion is very limited since (1) a negative ion must necessarily be accompanied by at least one positive ion and (2) the hydrogen ion can only carry a single positive charge. The additional information provided by coincidence measurements allows us to revisit and discuss previous results concerning negative ion formation.

Both the $\mathrm{H}^{-}$and $\mathrm{O}^{-}$NIY have been shown by Stolte et al. ${ }^{8}$ to have interesting trends just above threshold. For $\mathrm{O}^{-}$NIY, the slow exponential decay has been attributed to electron recapture. Both the $\mathrm{O}^{-} / \mathrm{H}^{+}$and $\mathrm{O}^{-} / \mathrm{H}^{+} / \mathrm{H}^{+}$yields in our experiment match well this behavior, and confirms that for $\mathrm{O}^{-}$production $\mathrm{H}_{2} \mathrm{O}^{*+} \rightarrow \mathrm{O}^{-}+\mathrm{H}^{+}+\mathrm{H}^{+}$fragmentation is the main mechanism. For $\mathrm{H}^{-}$NIY, Stolte et al. noted a step-like increase above O 1s IP and the yield settled at a higher level than below IP. In our experiments, the channels involving $\mathrm{H}^{-} ; \mathrm{H}^{-} / \mathrm{H}^{+}, \mathrm{H}^{-} / \mathrm{O}^{+}, \mathrm{H}^{-} / \mathrm{O}^{2+}$ and $\mathrm{H}^{-} / \mathrm{O}^{+} / \mathrm{H}^{+}$; also retains intensity above threshold and $\mathrm{H}^{-} / \mathrm{H}^{+}$exhibits a strong increase. There are two channels which can produce $\mathrm{H}^{-} / \mathrm{H}^{+}$ following O 1s ionization: Auger decay,

$$
\begin{gathered}
\mathrm{H}_{2} \mathrm{O}^{+* *} \rightarrow \mathrm{H}_{2} \mathrm{O}^{2+*}+e_{\text {Auger }}^{-} \\
\mathrm{H}_{2} \mathrm{O}^{2+*} \rightarrow \mathrm{H}^{-}+\mathrm{O}^{2+}+\mathrm{H}^{+},
\end{gathered}
$$

where ${ }^{*}$ indicates a valence-excited species; or radiative decay,

$$
\begin{gathered}
\mathrm{H}_{2} \mathrm{O}^{+* *} \rightarrow \mathrm{H}_{2} \mathrm{O}^{+*}+h \nu_{\text {fluorescence }} \\
\mathrm{H}_{2} \mathrm{O}^{+*} \rightarrow \mathrm{H}^{-}+\mathrm{O}^{+}+\mathrm{H}^{+} .
\end{gathered}
$$

Stolte et al. suggested that $\mathrm{H}^{-}$ions above IP originated from decay of doubly charged parent molecules decaying to $\mathrm{H}^{-} / \mathrm{O}^{2+} / \mathrm{H}^{+}$. Indeed, we find that the $\mathrm{H}^{-} / \mathrm{O}^{2+}$ yield is stronger above 
threshold in relative terms, but it does not display a similar increase as $\mathrm{H}^{-} / \mathrm{H}^{+}$above IP. We also find that $\mathrm{H}^{-} / \mathrm{O}^{+}$retains intensity, which must originate either from a singly charged or a neutral $\mathrm{H}_{2} \mathrm{O}$ parent molecule. However, since the strong increase in $\mathrm{H}^{-} / \mathrm{H}^{+}$yield is not reflected in other yields, it should result from the non-resonant radiative decay ${ }^{5}$ above threshold.

The most striking feature above the O 1s IP (Fig. 4) is the broad peak in the $\mathrm{H}^{-} / \mathrm{O}^{+}$yield with maximum at $\sim 556 \mathrm{eV}$ which is not reflected in other NIPICO channels. Earlier studies have shown an increase of the $\mathrm{H}_{2} \mathrm{O}$ photoabsorption cross section at this photon energy, ${ }^{24,25}$ which has been attributed to core-valence doubly excited states similar to the structure at $550 \mathrm{eV}$. Also, the maximum of the observed feature roughly coincides with the first shake-up limit at $556.75 \mathrm{eV}$, attributed to a $1 a_{1}^{-1} 3 a_{1}^{-1} 4 a_{1}^{1}$ state. $\mathrm{A} \mathrm{H}^{-} / \mathrm{O}^{+}$pair can only be created from a neutral or singly charged parent molecule. It is probable that the increased yield originates from doubly excited states of type $1 a_{1}^{-1} \mathrm{val}^{-1}$ virt $^{2}$ decaying by Auger emission and fragmenting to $\mathrm{H}^{-} / \mathrm{O}^{+} / \mathrm{H}^{+}$. While radiative decay from core-ionized shake-up states could contribute to the $\mathrm{H}^{-} / \mathrm{O}^{+}$yield, that non-resonant channel would not be expected to give rise to a peak. Radiative decay can thus be excluded as a viable production mechanism for the $\mathrm{H}^{-} / \mathrm{O}^{+}$peak at $\sim 556 \mathrm{eV}$.

A broad resonance in the yield of $\mathrm{O}^{-}$has been observed around $550 \mathrm{eV}$ photon energy, far above the O 1s IP $;^{8}$ other negative and positive yields were unaffected. Auger spectroscopy has revealed that excited states embedded at $550 \mathrm{eV}$ undergo ultra-fast dissociation and were identified as core--valence doubly excited neutral states (i.e. where the absorption of a single photon simultaneously promotes both a core and a valence electron to virtual orbitals). ${ }^{26}$ While our data show a small increase in the $\mathrm{O}^{-} / \mathrm{H}^{+}$yield above $550 \mathrm{eV}$ it does not appear as a pronounced peak. The cause for the observed difference between our data and the NIYs measured by Stolte et al. can not be conclusively determined, but it could be speculated that our NIPICO yield suffer from a large loss of high-energy $\mathrm{H}^{+}$ions created in the $\mathrm{O}^{-} / \mathrm{H}^{+} / \mathrm{H}^{+}$ channel. 
It should be possible to assess the contribution of radiative decay below the $\mathrm{O}$ 1s IP by comparing NIPICO and NIPIPICO channels: if they show identical behavior, the NIPICO events are simply a subset of the NIPIPICO events, and originate from the same (nonradiative) mechanism. We observe that the $\mathrm{O}^{-} / \mathrm{H}^{+}$and $\mathrm{O}^{-} / \mathrm{H}^{+} / \mathrm{H}^{+}$yields are not quite similar below IP (Fig. 2). In particular, the ratio between intensities at the $4 a_{1}$ and $2 b_{2}$ resonances is $0.32 \pm 0.06$ for the $\mathrm{O}^{-} / \mathrm{H}^{+}$yield, but only $0.21 \pm 0.06$ for the $\mathrm{O}^{-} / \mathrm{H}^{+} / \mathrm{H}^{+}$yield. While this $50 \%$ difference is within the combined margin of error of our measurement, any significant reduction of the $\mathrm{O}^{-} / \mathrm{H}^{+} / \mathrm{H}^{+}$yield compared to $\mathrm{O}^{-} / \mathrm{H}^{+}$could suggest radiative decay, although we did not observe any two-body ion fragmentation channels $-\mathrm{OH}^{-} / \mathrm{H}^{+}$, $\mathrm{H}^{-} / \mathrm{OH}^{+}$or $\mathrm{O}^{-} / \mathrm{H}_{2}^{+}-$uniquely originating from a neutral parent molecule. The $\mathrm{O}^{-}$fragment can only be produced via the neutral three-body dissociation pathway

$$
\begin{array}{r}
\mathrm{H}_{2} \mathrm{O}^{* *} \rightarrow \mathrm{H}_{2} \mathrm{O}^{*}+h \nu_{\text {fluorescence }} \\
\mathrm{H}_{2} \mathrm{O}^{*} \rightarrow \mathrm{O}^{-}+\mathrm{H}^{+}+\mathrm{H}
\end{array}
$$

or from singly charged parent ion states

$$
\begin{array}{r}
\mathrm{H}_{2} \mathrm{O}^{* *} \rightarrow \mathrm{H}_{2} \mathrm{O}^{+*}+e_{\text {Auger }}^{-} \\
\mathrm{H}_{2} \mathrm{O}^{+*} \rightarrow \mathrm{O}^{-}+\mathrm{H}^{+}+\mathrm{H}^{+} .
\end{array}
$$

In this case, the difference between the NIPICO and NIPIPICO channels would originate from additional channel producing $\mathrm{O}^{-} / \mathrm{H}^{+}$ion pair, i.e. the radiative decay. Another possibility is that our spectrometer do not accept all the $\mathrm{H}^{+}$ions. It is expected that light $\mathrm{H}^{+}$ carries most of the kinetic energy in three-body breakups, and more of them would escape detection with higher KER. From peak-shape analysis we can conclude that the KER is slightly larger at the $4 a_{1}$ resonance than at $2 b_{2}$, although with fairly small KER at both of them. These combined observations suggest that the radiative decay contribution below IP is small (or non-existing), but its role can not be conclusively determined from our study. 


\section{Conclusion}

We have presented NIPICO and NIPIPICO yields at the $\mathrm{O} 1 s$ edge of $\mathrm{H}_{2} \mathrm{O}$. Our results complement earlier measurements of non-coincident negative-ion yields ${ }^{8}$ and coincident positive-ion yields ${ }^{7}$ in this energy region. $\mathrm{O}^{-}$observed in coincidence with $\mathrm{H}^{+}$ions was the strongest observed NIPI(PI)CO channel. $\mathrm{H}^{-}$was observed in coincidence with $\mathrm{H}^{+}, \mathrm{O}^{+}$ and $\mathrm{O}^{2+}$. NIPICO channels are observed both below and above the O $1 s$ IP. Fragmentation following (resonant) Auger decay of core-hole states is the main pathway to the production of negative ions, but we have also observed evidence that the radiative decay of core-hole states contributes to the negative ion production above the O 1s IP. An increase in the $\mathrm{H}^{-} / \mathrm{O}^{+}$ yield around $556 \mathrm{eV}$ is attributed to non-radiative decay of doubly excited states.

The data show that our recently commissioned negative-ion/positive-ion coincidence spectrometer can be readily utilized to disentangle unknown decay and dissociation processes involving negative-ion production. Negative ion production, which is typically orders of magnitude less probable than that of positive ions provides a very sensitive probe for fragmentation studies. We envision that in the future the instrument can become a valuable tool to chart fragmentation patterns of polyatomic molecules.

\section{Acknowledgement}

The authors thank Claudiu Bulbucan for doing electrostatical simulations of the instrument acceptance. We acknowledge Elettra-Sincrotrone for providing beamtime (proposal number 20145053) and the staff at Elettra for their assistance during commissioning of the instrument. The research leading to these results has received funding from the European Community's Seventh Framework Programme (FP7/2007-2013) under grant agreement n:o 312284. 


\section{References}

(1) Nilsson, A.; Pettersson, L. G. M. The structural origin of anomalous properties of liquid water. Nat. Comm. 2015, 6, 8998.

(2) Bartels-Rausch, T.; Bergeron, V.; Cartwright, J. H. E.; Escribano, R.; Finney, J. L.; Grothe, H.; Gutiérrez, P. J.; Haapala, J.; Kuhs, W. F.; Pettersson, J. B. C. et al. Ice structures, patterns, and processes: A view across the icefields. Rev. Mod. Phys. 2012, $84,885-944$.

(3) Siegbahn, H.; Asplund, L.; Kelfve, P. The Auger electron spectrum of water vapour. Chem. Phys. Lett. 1975, 35, 330-335.

(4) Nordgren, J.; Werme, L. P.; Ågren, H.; Nordling, C.; Siegbahn, K. The X-ray emission spectrum of water. J. Phys. B: At. Mol. Phys. 1975, 8, L18.

(5) Kashtanov, S.; Augustsson, A.; Luo, Y.; Guo, J.-H.; Såthe, C.; Rubensson, J.-E.; Siegbahn, H.; Nordgren, J.; Ågren, H. Local structures of liquid water studied by x-ray emission spectroscopy. Phys. Rev. B 2004, 69, 024201.

(6) Hjelte, I.; Piancastelli, M.; Fink, R.; Björneholm, O.; Bässler, M.; Feifel, R.; Giertz, A.; Wang, H.; Wiesner, K.; Ausmees, A. et al. Evidence for ultra-fast dissociation of molecular water from resonant Auger spectroscopy. Chem. Phys. Lett. 2001, 334, 151-158.

(7) Piancastelli, M. N.; Hempelmann, A.; Heiser, F.; Gessner, O.; Rüdel, A.; Becker, U. Resonant photofragmentation of water at the oxygen $\mathrm{K}$ edge by high-resolution ionyield spectroscopy. Phys. Rev. A 1999, 59, 300-306.

(8) Stolte, W. C.; Sant'Anna, M. M.; Öhrwall, G.; Dominguez-Lopez, I.; Piancastelli, M. N.; Lindle, D. W. Photofragmentation dynamics of core-excited water by anion-yield spectroscopy. Phys. Rev. A 2003, 68, 022701. 
(9) Berkowitz, J. Atomic and Molecular Photoabsorption: Partial Cross Sections; Elsevier Science, 2015.

(10) Hunniford, C. A.; Scully, S. W. J.; Dunn, K. F.; Latimer, C. J. Fragment anion spectroscopy of water in the inner and outer valence regions. J. Phys. B: At., Mol. Opt. Phys. 2007, 40, 1225.

(11) Stolte, W. C.; Hansen, D. L.; Piancastelli, M. N.; Dominguez Lopez, I.; Rizvi, A.; Hemmers, O.; Wang, H.; Schlachter, A. S.; Lubell, M. S.; Lindle, D. W. Anionic Photofragmentation of CO: A Selective Probe of Core-Level Resonances. Phys. Rev. Lett. 2001, $86,4504-4507$.

(12) Öhrwall, G.; Sant'Anna, M. M.; Stolte, W. C.; Dominguez-Lopez, I.; Dang, L. T. N.; Schlachter, A. S.; Lindle, D. W. Anion and cation formation following core-level photoexcitation of CO 2. J. Phys. B: At., Mol. Opt. Phys. 2002, 35, 4543.

(13) Stolte, W. C.; Öhrwall, G.; Sant'Anna, M. M.; Lopez, I. D.; Dang, L. T. N.; Piancastelli, M. N.; Lindle, D. W. $100 \%$ site-selective fragmentation in core-holephotoexcited methanol by anion-yield spectroscopy. J. Phys. B: At. Mol. Opt. Phys. 2002, 35, L253-L259.

(14) Yu, S.-W.; Stolte, W. C.; Öhrwall, G.; Guillemin, R.; Piancastelli, M. N.; Lindle, D. W. Anionic and cationic photofragmentation of core-excited $\mathrm{N}_{2} \mathrm{O}$. J. Phys. B: At., Mol. Opt. Phys. 2003, 36, 1255.

(15) Piancastelli, M. N.; Stolte, W. C.; Guillemin, R.; Wolska, A.; Yu, S.-W.; Sant'Anna, M. M.; Lindle, D. W. Anion and cation-yield spectroscopy of core-excited SF6. J. Chem. Phys. 2005, 122, 094312.

(16) Scully, S. W. J.; Mackie, R. A.; Browning, R.; Dunn, K. F.; Latimer, C. J. Negative photoion spectroscopy of SF6 in the inner valence and S 2p energy regions. J. Phys. B: At., Mol. Opt. Phys. 2002, 35, 2703. 
(17) Piancastelli, M.; Guillemin, R.; Stolte, W.; Ceolin, D.; Lindle, D. Partial cation and anion-yield experiments in ammonia around the $\mathrm{N}$ 1s ionization threshold. J. Electron Spectrosc. Relat. Phenom. 2007, 155, 86 - 90.

(18) Yu, S.-W.; Stolte, W. C.; Guillemin, R.; Öhrwall, G.; Tran, I. C.; Piancastelli, M. N.; Feng, R.; Lindle, D. W. Photofragmentation study of core-excited NO. J. Phys. B: At., Mol. Opt. Phys. 2004, 37, 3583.

(19) Rühl, E.; Flesch, R. Mechanism of anion formation in C $1 s \rightarrow \pi^{*}-$ excited carbon dioxide. J. Chem. Phys. 2004, 121, 5322-5327.

(20) Stråhlman, C.; Sankari, R.; Kivimäki, A.; Richter, R.; Coreno, M.; Nyholm, R. A Tandem Time-of-Flight Spectrometer for Negative-Ion/Positive-Ion Coincidence Measurements with Soft X-ray Excitation. Rev. Sci. Instrum. 2016, 87, 013109.

(21) Blyth, R.; Delaunay, R.; Zitnik, M.; Krempasky, J.; Krempaska, R.; Slezak, J.; Prince, K.; Richter, R.; Vondracek, M.; Camilloni, R. et al. The high resolution Gas Phase Photoemission beamline, Elettra. J. Electron Spectrosc. Relat. Phenom. 1999, 101-103, 959-964.

(22) Prince, K. C.; Blyth, R. R.; Delaunay, R.; Zitnik, M.; Krempasky, J.; Slezak, J.; Camilloni, R.; Avaldi, L.; Coreno, M.; Stefani, G. et al. The gas-phase photoemission beamline at Elettra. J. Synchrotron Rad. 1998, 5, 565-568.

(23) Morin, P.; Nenner, I.; Guyion, P. M.; Dutuit, O.; Ito, K. Time of flight photoelectron spectroscopy using synchrotron radiation study of resonances in $\mathrm{O}_{2}$. J. Chim. Phys. 1980, 77, 605-612.

(24) Wight, G. R.; Brion, C. E. K-shell excitation of $\mathrm{CH}_{4}, \mathrm{NH}_{3}, \mathrm{H}_{2} \mathrm{O}, \mathrm{CH}_{3} \mathrm{OH}, \mathrm{CH}_{3} \mathrm{OCH}_{3}$ and $\mathrm{CH}_{3} \mathrm{NH}_{2}$ by $2.5 \mathrm{keV}$ electron impact. J. Electron Spectrosc. Relat. Phenom. 1974, $4,25-42$. 
(25) Kivimäki, A.; de Simone, M.; Coreno, M.; Feyer, V.; Melero García, E.; Álvarez Ruiz, J.; Richter, R.; Prince, K. C. Observation of core-hole double excitations in water using fluorescence spectroscopy. Phys. Rev. A 2007, 75, 014503.

(26) Piancastelli, M. N.; Sankari, R.; Sorensen, S.; De Fanis, A.; Yoshida, H.; Kitajima, M.; Tanaka, H.; Ueda, K. Resonant Auger decay of above-threshold core-excited $\mathrm{H}_{2} \mathrm{O}$. Phys. Rev. A 2005, 71, 010703.
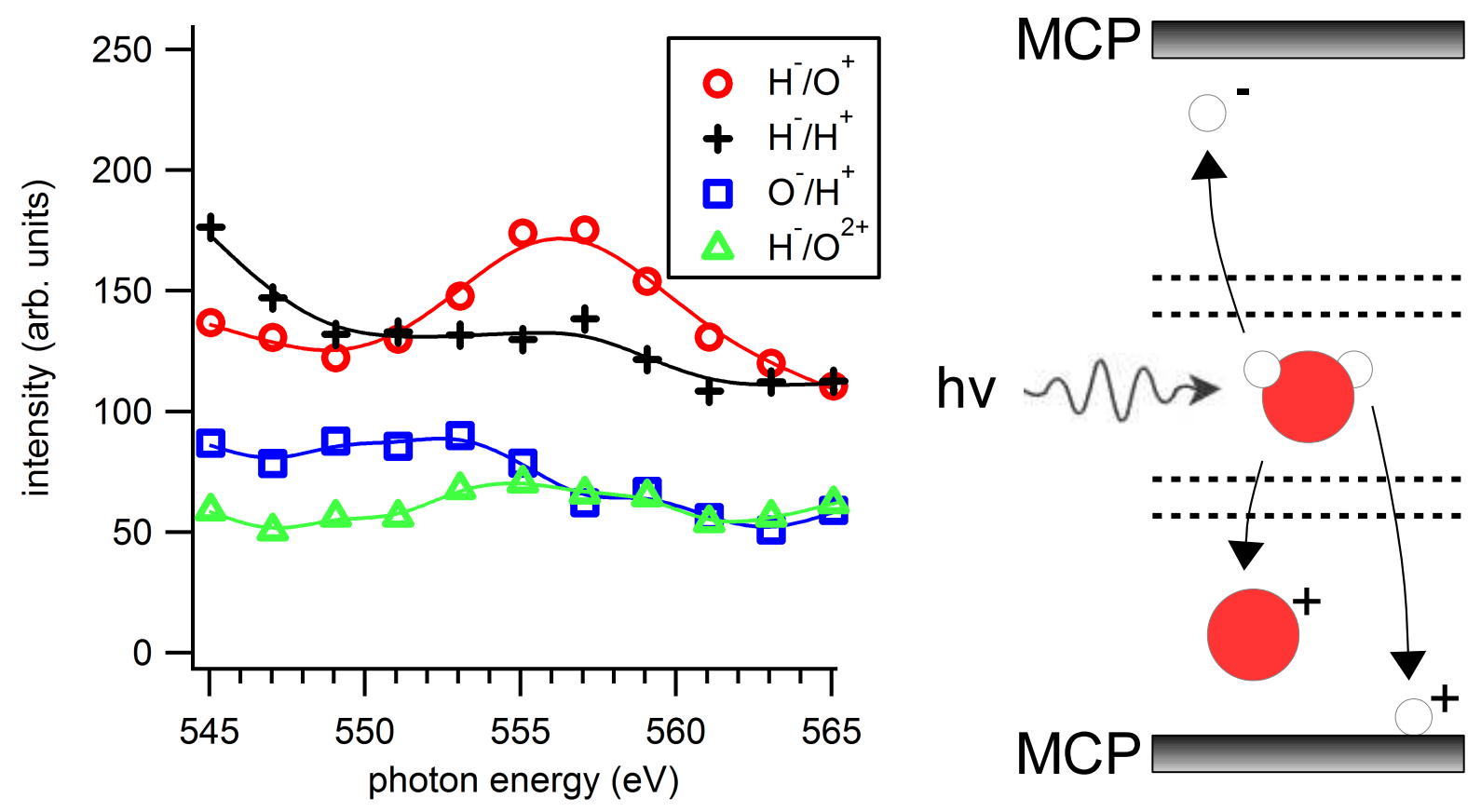